Vol. 5, No. 02; 2021

ISSN: 2581-3366

\title{
The Roles of SDF-1/CXCR4, MCP-1/CCR2 and CCL5/CCR5 Chemokine Pathway in Hypertrophic Scarring
}

\author{
Authors \\ ${ }^{1}$ st Author Name: Hirokatsu Umeyama, MD \\ Wound Healing Research Group, Department of Surgery, University of Alberta, Edmonton, \\ Alberta, Canada. \\ Department of Plastic and Reconstructive Surgery, Graduate School of Medicine, Tohoku \\ University, Sendai, Miyagi, Japan. \\ ${ }^{2}$ nd Author Name: Jie Ding, MD, PhD \\ Wound Healing Research Group, Department of Surgery, University of Alberta, Edmonton, \\ Alberta, Canada. \\ ${ }^{3}$ rd Author Name: Antoinette Nguyen, PhD. \\ Wound Healing Research Group, Department of Surgery, University of Alberta, Edmonton, \\ Alberta, Canada. \\ ${ }^{4}$ th Author Name: Servet Elcin Alpat, MD. \\ ${ }^{4}$ th Author Affiliation: \\ Wound Healing Research Group, Department of Surgery, University of Alberta, Edmonton, \\ Alberta, Canada. \\ ${ }^{5}$ th Author Name: Josue R Silva, MD, MSc. \\ Wound Healing Research Group, Department of Surgery, University of Alberta, Edmonton, \\ Alberta, Canada. \\ ${ }^{6}$ th Author Name: Peter Kwan, MD, PhD. \\ Division of Plastic and Reconstructive Surgery and Wound Healing Research Group, \\ Department of Surgery, University of Alberta, Edmonton, Alberta, Canada. \\ ${ }^{7}$ th Author Name: Munetomo Nagao, MD, PhD. \\ Department of Plastic and Reconstructive Surgery Graduate School of Medicine Tohoku \\ University, Sendai, Miyagi, Japan. \\ $8_{\text {th }}$ Author Name: Masahiro Tachi, MD, PhD. \\ Department of Plastic and Reconstructive Surgery, Graduate School of Medicine, Tohoku \\ University, Sendai, Miyagi, Japan. \\ ${ }^{9}$ th Author Name: Edward E. Tredget, MD, MSc. FRCS(c). \\ Division of Plastic and Reconstructive Surgery, Division of Critical Care Medicine, and Wound \\ Healing Research Group, Department of Surgery, University of Alberta, Edmonton, Alberta, \\ Canada.
}




\title{
International Journal of Medical Science and Health Research
}

Vol. 5, No. 02; 2021

ISSN: $2581-3366$

Corresponding authors

Jie Ding, MD, PhD

Wound Healing Research Group

Department of Surgery

161 HMRC, University of Alberta

Phone: 1-780-4920061

Edward E Tredget, MD, MSc, FRCS(c)

Division of Plastic Surgery, Department of Surgery

2D2.28 WMC, University of Alberta Hospital

Edmonton, AB, T6G2S2. Canada

Phone: 1-780-4076979

doi: 10.51505/ijmshr.2021.5215

URL: http://dx.doi.org/10.51505/ijmshr.2021.5215

\begin{abstract}
Hypertrophic scars are a dermal form of fibroproliferative diseases that develop after skin trauma to the deep dermis. Dysfunction of the chemokine network can result in prolonged inflammation, abnormal blood vessel development, and a fibrotic wound-healing environment. To understand the role of the chemokine signaling in pathological scar formation, the inhibitory effects of scar formation by chemokine inhibitors of AMD3100, Maraviroc, and CAS445479-97-0 were investigated using a mouse model of dermal fibrosis, in which human split-thickness skin grafts were transplanted into full-thickness excision wounds in the dorsal of athymic nude mice, and the human skin grafts developed scars morphologically and histologically similar to human hypertrophic scars. The mice were treated with one of chemokine inhibitors from 5 days after grafting daily for 1 week and thereafter once a week. Mouse wounds were monitored, and scar tissues were collected at 2, 6, and 12 weeks after grafting for further histological and biochemical analysis. AMD3100 and Maraviroc reduced wound contraction. AMD3100 improved collagen orientation and up-regulated mRNA level of decorin in the early phase of wound healing. AMD3100, CAS445479-97-0 and Maraviroc reduced scar thickness, macrophage recruitment and myofibroblast formation, and down-regulated gene expressions of type I collagen in the early phase, heat shock protein 47 in the late phase, alpha-smooth muscle actin, and connective tissue growth factor throughout the whole phase of wound healing. Blocking chemokine pathways significantly improved scars in this dermal fibrotic model, which can be potential therapeutic targets for hypertrophic scars.
\end{abstract}

Keywords: Hypertrophic scars, CXCR4, CCR2, CCR5, nude mouse model of dermal fibrosis

\section{Introduction}

Hypertrophic scars (HTS) are a dermal form of fibroproliferative disorders that develop after skin injuries into the deep dermis such as burns and surgeries. HTS are defined as visible, raised scars that do not spread into surrounding tissues. Contracture deformities, aesthetic disfigurement, and long hospitalization and rehabilitation frequently occur in patients, which 


\section{International Journal of Medical Science and Health Research}

Vol. 5, No. 02; 2021

ISSN: 2581-3366

may lead to psychological complications (1-2). Various therapies for HTS have been identified, however, the optimal therapy has not yet been developed.

Cellular and molecular factors, such as chemokines, bone marrow-derived stem cells, transforming growth factor-beta (TGF- $\beta$ ), fibroblast heterogeneity, toll-like receptors, and small leucine-rich proteoglycans play important roles in wound healing and pathological scar formation. Especially chemokines are involved in all stages of wound healing, hemostasis, inflammation, proliferation, and remodeling. Hyperactivity of chemokines results in pathological scarring. A deeper understanding of the pathways can help to develop therapeutic strategies for HTS that are more effective.

Wound healing involves complex interactions within the basal epidermis, appendages, and underlying mesenchyme between keratinocytes, fibroblasts, extracellular matrix (ECM), and stem cells (3). A variety of molecules, including chemokines, growth factors, and cytokines organize and regulate these interactions.

The roles of chemokines in wound healing processes, as well as acquired immunodeficiency syndrome caused by human immunodeficiency viruses-1 (HIV-1), inflammatory bowel diseases, cardiovascular diseases, atherosclerosis, rheumatoid arthritis, and cancer have been mentioned in many studies in recent years. Chemokines are a family of tiny 8-10 KDa chemotactic cytokines which have been categorized into four primary subfamilies on the basis of the arrangement of the two N-terminal cysteine residues: C (XCL), CC (CCL), CXC (CXCL), and CX3C (CX3CL) chemokines. In several fundamental biological processes, they play critical roles, such as leukocyte trafficking and homing, tumorigenesis and metastasis, inflammation, autoimmune response, and viral infection.

Numerous blood-borne cells regulate normal wound healing procedures and recruitment of these cells into tissues is tightly managed by chemokine pathways, which are essential modulators in wound healing at each phase (4-6). Dysregulation of the chemokine pathways can lead to prolonged inflammation, the development of dysregulated vessels, and chronic environment that result in various forms of fibrosis such as HTS, keloids, scleroderma, and psoriasis (7). Histologically, owing to elevated numbers of dermal fibroblasts and myofibroblasts, and recruited peripheral hematopoietic cells, HTS is hypercellular. Blood-borne cell recruitment indicates that chemokine pathways play an important role in HTS formation (5).

Stromal cell-derived factor-1 (SDF-1), also called CXCL12, is expressed in the epidermis, dermis, hair follicles, blood vessels, and sweat glands. Hematopoietic progenitor cell trafficking between the peripheral circulation and bone marrow is regulated by SDF-1. SDF-1 binds to its receptor, CXCR4 on endothelial cells, and induces chemotaxis of endothelial cells, which is essential for vascularization. SDF-1 is involved in the homing of circulating cells via CXCR4 during wound repair for skin, skeleton, myocardium, vessel, and lung (5).

By facilitating the migration of CD14+CXCR4+ cells from the bloodstream to wound sites, where they can differentiate into fibrocytes and myofibroblasts, the SDF-1/CXCR4 pathway is also involved in HTS formation (8). After the injury, local dermal fibroblasts are triggered by inflammatory signals and produce SDF-1, which attracts CXCR4-expressing monocytes from the systemic circulation to the wound site. In burn patients, the SDF-1/CXCR4 pathway is upregulated during wound healing and remains up-regulated in HTS tissue after wound healing as 


\section{International Journal of Medical Science and Health Research}

Vol. 5, No. 02; 2021

ISSN: 2581-3366

opposed to normal skin (8). Our group has recorded that blocking the SDF-1/CXCR4 pathway by CTCE-9908, an analog of SDF-1, minimized HTS formation in the mouse model by reducing the aggregation of macrophages and myofibroblasts, improving the remodeling of collagen fibers, and down-regulating the gene and protein expression of fibrotic growth factors (9).

Monocyte chemoattractant protein-1 (MCP-1) also known as CCL2, is developed by several types of cells, including fibroblasts, endothelial, epithelial, smooth muscle, mesangial, astrocytic, monocytic, and microglial cells. MCP-1 is released by neutrophils and monocytes themselves at the early stages of wound healing, and keratinocytes at later stages of the healing process (10). Monocyte chemotaxis to active inflammatory sites in the wound healing process is regulated by MCP-1 and monocytes differentiate into macrophages (11). Macrophages phagocytose apoptotic neutrophils and other dead cells and secrete cytokines, growth factors, and chemokines that promote inflammation and wound repair at the later stage of healing process.

MCP-1 binds to its receptor CCR2 on endothelial cells, and by regulating membrane-type 1matrix metalloproteinase, the MCP-1/CCR2 pathway mediates neovascularization and promotes fibroplasia and ECM production $(12,13)$. MCP-1 knockout mice develop minimal skin scars after mechanical force, indicating that the inflammatory chemokine pathway involved in the skin fibrosis induced by mechanical force occurs through focal adhesion kinase (14).

CCL5, also known as RANTES (regulated upon activation, normal $\mathrm{T}$ cell expressed and secreted), binds to its receptor, CCR5 expressed on the surface of several immune cells such as macrophages, monocytes, microglia, dendritic cells, and active memory CD4 T cells. CCL5 enhances production of vascular endothelial growth factors in human osteosarcoma cells and promotes angiogenesis (15). The recruitment of endothelial progenitor cells to the wound site is regulated by CCR5. In mice, gene expression of CCL5 and CCR5 are upregulated at wound sites where the CCR5 protein is detected exclusively in macrophages and endothelial cells. CCR5 deficiency decreased the recruitment of endothelial progenitor cells and reduced neovascularization and collagen production in wounds leading to delayed wound healing (16). In this study, we evaluate the effects of blocking these chemokine pathways on scar formation by their receptor inhibitors using a mouse model of dermal fibrosis.

\section{Materials and Methods}

\subsection{Animals}

Athymic nude mice, male, Foxn-1 (-/-), and 4 weeks of age were purchased from the Jackson Laboratory (Bar Harbor, ME) and preconditioned for 2 weeks in the animal facility of University of Alberta. The standardized operating procedures and animal care were approved by the University of Alberta Animal Care and Use Committee.

2.2 Preparation of human split-thickness skin grafts

Normal skin tissues were obtained from female patients who underwent cosmetic abdominoplasty following informed written consent. Split-thickness skin grafts (STSGs) were harvested from them using an electric dermatome (Padgett Instruments, Inc., Kansas City, MO) set at $0.03 \mathrm{~cm}$, thereafter, the harvested grafts were cut into $2.0 \mathrm{~cm} \times 1.5 \mathrm{~cm}$ pieces. 


\section{International Journal of Medical Science and Health Research}

Vol. 5, No. 02; 2021

ISSN: 2581-3366

2.3 Establishment of a mouse model of dermal fibrosis

A full-thickness skin $(2.0 \mathrm{~cm} \times 1.5 \mathrm{~cm})$ at the dorsum of each mouse was excised under general anesthesia to create a wound as previously described (17-18). Human STSG was then transplanted onto the wound and sutured with 4-0 silk sutures. Tie-over bolus dressing was performed using a fine mesh lubricated gauze (Xeroform, Covidien, Mansfield, MA) and gauze to protect and attach the grafts to the edges of the wound. The dressing was removed 1 week after grafting.

\subsection{Treatment of mice and collection of scar biopsies}

The mice were treated from 5 days after grafting daily for 1 week and thereafter once a week by subcutaneously injecting AMD3100 (6 mg/kg) (19-20), or CAS445479-97-0 (0.5 mg/kg) (21), or Maraviroc $(10 \mathrm{mg} / \mathrm{kg})$ in $100 \mu \mathrm{L}$ phosphate-buffered saline (PBS) (22), or $100 \mu \mathrm{L}$ PBS as vehicle control around the perimeter of the wounds. The mice were euthanized at 2, 6, and 12 weeks postoperatively. $0.5-1.0 \mathrm{ml}$ of peripheral blood was collected by cardiac puncture under general anesthesia and suspended in EDTA (ethylenediaminetetraacetic acid) tube. For the biochemical and histological study, scars (xenografts) were excised and collected from the mice. AMD3100 is a small bicyclic molecule that was originally identified as a selective CXCR4 inhibitor with anti-HIV-1 activity. It inhibits the interaction between CXCR4 on CD34+ hematopoietic stem cells and SDF-1 on bone marrow stromal cells, essentially blocking the chemotactic actions of SDF-1 (23). AMD3100 was purchased from AdooQ Bioscience (Irvine, CA).

CAS 445479-97-0 has a glycinamide/3-trifluoromethyl benzamide moiety that is prevalent throughout the CCR2 antagonist field (24). It is CCR2 antagonist, which was purchased from Millipore Sigma (Burlington, MA).

Maraviroc is an inhibitor that prevents entry of CCR5-tropic virus into T-cells and therefore is approved for the treatment of R5-tropic patients with acquired immunodeficiency syndrome (25). Maraviroc was purchased from AdooQ Bioscience (Irvine, CA).

\subsection{Digital photography}

For recording wound healing and scar formation, digital photographs were taken with a ruler once a week. Wound areas were measured using ImageJ software (National Institutes of Health, Bethesda, MD).

2.6 Hematoxylin and Eosin (H\&E) staining for scar thickness and vascularity in the xenografts Paraffin-embedded scar sections, which biopsied from the mice treated with AMD3100, CAS445479-97-0, Maraviroc or the vehicle (PBS) at 2, 6, and 12 weeks after grafting, were deparaffinized, rehydrated through five of decreasing gradient ethanol, and immersed in distilled water for 2 minutes before incubating them in hematoxylin solution for 1 minute at room temperature. The sections were dipped in distilled water and acid alcohol, washed by running tap water for 5 minutes, and rinsed in $95 \%$ ethanol for 1 minute. Thereafter the sections were counterstained in eosin solution for 2 minutes at room temperature, dehydrated in $95 \%$ ethanol and two changes of $100 \%$ ethanol, and cleared in xylene. The sections were then mounted with permount (Fisher Scientific, Hampton, NH) and covered with glass coverslips. 


\section{International Journal of Medical Science and Health Research}

Vol. 5, No. 02; 2021

ISSN: 2581-3366

Under the bright field microscope, $H \& E$ staining images were taken to evaluate scar thickness and vascularity. The epidermal thickness and the dermal thickness were measured in three locations (both edges and middle of the biopsies) for each sample by ImageJ software. The epidermal thickness was determined as the distance from epidermal surface to the basement membrane, and the dermal thickness as the distance between the epidermal-dermal junction and the dermal-adipose layer junction. The number of blood vessels in 15 high-power fields (HPFs) of the dermis (from top to bottom of dermis) was counted to evaluate the cellularity and vascularity.

2.7 Masson's Trichrome staining for collagen deposition

Paraffin-embedded scar sections were deparaffinized, rehydrated, and mordant fixed in Bouin's solution before staining in Weigert's iron hematoxylin, Biebrich scarlet acid, and phosphomolybdic-phosphotungstic acid solution for 5 minutes each. The sections were transferred to aniline blue and stained for 5 minutes further. After differentiating in $1 \%$ acetic acid for 2 minutes and rinsing by distilled water, the sections were dehydrated in alcohol, cleared in xylene, mounted with permount (Fisher Scientific, Hampton, NH), and covered with glass coverslips.

Masson's Trichrome staining is a 3-color histology staining, by which collagen fibers are visualized in blue, nuclei in black, and keratin in red under the bright field microscope. Masson's trichrome staining images were taken under the bright field microscope to observe collagen deposition in the dermis for each sample.

2.8 Picrosirius red staining for collagen orientation

Paraffin-embedded scar sections were deparaffinized and rehydrated through five decreasing gradients of ethanol before incubating them in Picrosirius Red (abcam®), Cambridge, UK) solution for 1 hour at room temperature. Thereafter, the sections were washed by acetic acid solution twice, dehydrated in $100 \%$ ethanol two times, and cleared in xylene. The sections were mounted with permount (Fisher Scientific, Hampton, NH), covered with glass coverslips, and then examined using a polarizing microscope (Nikon ECLIPSE Ti; Nikon instruments Inc., Melville, NY) in order to visualize collagen fibers. From each slide, five images were taken at three areas, both edges and middle of the biopsies. The first image was in the most superficial dermal layer, directly underneath the epidermis, the second image was directly underneath the first image, the third image was in the middle of the dermis, the fourth image was directly underneath the third image, and the fifth image was at the border of the dermis and fatty tissue, at the deepest level of the dermis.

Image analysis was performed using the power-plot generated by means of the Fast Fourier Transform (FFT) feature in ImageJ software which has been used to estimate the power spectrum of images and the collagen orientation index (COI) was calculated by determining stretch or elongation of the FFT spectrum using the equation: $\mathrm{COI}=1-$ (width/height). This analysis results in values that range from 0 to 1 , where 0 represents perfectly randomly oriented collagen fibers and 1 represents perfectly parallel-oriented collagen fibers (26-27).

www.ijmshr.com

Page 186 


\section{International Journal of Medical Science and Health Research}

Vol. 5, No. 02; 2021

ISSN: 2581-3366

2.9 Immunohistochemistry staining for myofibroblasts and macrophages

Paraffin-embedded scar sections were deparaffinized and rehydrated in five decreasing gradients of ethanol, and then treated for antigen retrieval with $0.05 \%$ trypsin for myofibroblast staining with alpha-smooth muscle actin ( $\alpha$-SMA) primary antibody and with sodium citrate buffer for macrophage staining with CD163 and F4/80 primary antibodies before permeabilization by $0.2 \%$ Triton-X in PBS for 20 minutes. Thereafter slides were blocked with $10 \%$ normal serum with $2 \%$ BSA in PBST for 1 hour. Then sections were incubated with primary antibody of rabbit anti- $\alpha$ SMA (Invitrogen, Waltham, MA), rat anti-F4/80 (Abcam, Cambridge, UK) and rabbit antiCD163 (Abcam, Cambridge, UK) diluted in PBST with $2 \%$ BSA overnight at $4^{\circ} \mathrm{C}$. The secondary antibody of goat anti-rabbit IgG (Dako. Santa Clara, CA) for $\alpha$-SMA and CD163 and rabbit anti-rat IgG (Dako. Santa Clara, CA) for F4/80 were applied at 1:500 dilution in PBST with $2 \%$ BSA for 30 minutes at room temperature. Avidin/biotin staining was performed using the Vectastain Elite ABC Kit (Vector Laboratories, Inc. Burlingame, CA) was applied for 30 minutes and washed with peroxidase substrate DAB (Vector Laboratories, Inc. Burlingame, CA) before counterstaining with hematoxylin. Dehydration through five increasing gradient ethanol was made before mounting the slides with permount (Fisher Scientific, Hampton, NH) and glass coverslips. Brown positively stained cells were counted in five HPFs under x200 magnification in all sections.

2.10 Immunofluorescence staining for decorin (DCN)

Following deparaffinization and rehydration, paraffin-embedded scar sections were treated for antigen retrieval by sodium citrate buffer. Slides in the staining dish containing sodium citrate buffer were incubated in the pre-heated water bath at $95-100{ }^{\circ} \mathrm{C}$ for 20 minutes, cooled down, and kept at room temperature for 20 minutes. Thereafter slides were permeabilized by $0.2 \%$ Triton-X in PBS for 20 minutes before blocking with 10\% goat serum with $2 \%$ BSA in PBST for 1 hour. Slides were then incubated with the primary antibody of rabbit anti-DCN IgG (Santa Cruz Biotechnology, Inc., Dallas, TX) for overnight followed by the secondary antibody of goat anti-rabbit IgG AF488 for 1 hour. After washing, slides were mounted with ProLong Gold antifade reagent with DAPI (Life Technologies, Carlsbad, CA), and covered. Fluorescence microscopy was performed by Nikon ECLIPSE Ti (Nikon instruments Inc., Melville, NY) and fluorescent images were taken at three areas (both edges and middle of the biopsies) for each sample. The density of DCN signal in the dermis (from top to bottom of dermis) of scars was calculated using ImageJ software.

2.11 Enzyme-linked immunosorbent assay (ELISA) for the quantity of DCN in the serum Peripheral blood was collected by cardiac puncture from the mice treated with AMD3100, CAS445479-97-0, Maraviroc, or PBS at 2, 6 and 12 weeks after grafting. Serum was isolated from peripheral blood and DCN was quantified by ELISA. Mouse DCN ELISA kit (R\&D Systems, Minneapolis, MN) was used according to the manufacturer's instructions. 


\section{International Journal of Medical Science and Health Research}

Vol. 5, No. 02; 2021

ISSN: 2581-3366

2.12 Quantitative polymerase chain reaction (qPCR) for the gene expression of fibrotic growth factors

Scars collected from the mice treated with AMD3100, CAS445479-97-0, Maraviroc, or PBS at 2, 6 and 12 weeks after grafting were powdered using Mikro Dismembrator (B. Braun Biotech, Allentown, PA) and then lysed using TRIzol reagent (Invitrogen, Carlsbad, CA). Total RNA was extracted using RNeasy Mini Kit (QIAGEN Sciences, Germantown, MD). A $0.5 \mu \mathrm{g}$ of total RNA was used for cDNA synthesis using cDNA Syntheses Kit (Invitrogen, Carlsbad, CA). The qPCR was conducted using Power SYBR Green PCR Master Mix (Applied Biosystems, Foster City, CA). The primers are 5'-CGAAGACATCCCACCAATCAC-3' (forward) and 5'TCATCGCACAACACCTTGC-3' (reve1rse) for type 1 collagen (COL-1), 5'AGCCCAGCCAAGCACTG-3' (forward) and 5'-CAAAGCCGGCCTTACAGAG-3' (reverse) for $\alpha$-SMA, 5'-ACCCACAACGAAATCTATGAC-3' (forward) and 5'GCTCCACTTTTAACTTGAGCC-3' (reverse) for TGF- $\beta 1$, 5'TGGAGATTTTGGGAGTACGG-3' (forward) and 5'-TACCAATGACAACGCCTCCT-3' (reverse) for connective tissue growth factor (CTGF), 5'-TGAAGAAGCTCTCCTACATCC-3' (forward) and 5'-AAACTCAATCCCAACTTAGCC-3' (reverse) for DCN, 5'TGGTGCGAGATAATCAGAGC-3' (forward) and 5'-CTTGTCAATGGCCTCAGTCA-3' (reverse) for heat shock protein 47 (HSP47), 5'-ACCCCTTCATTGACCTCAAC-3' (forward) and 5'-GAGATGATGACCCTTTTGGC-3' (reverse) for glyceraldehyde-3-phosphate dehydrogenase used as a normalization/internal control (Eurofins MWG Operon, Huntsville, AL). The StepOnePlus Real-Time PCR System (Applied Biosystems. Foster City, CA) was used to amplify and quantify cDNA. Relative gene expression was measured as cycle threshold $(\mathrm{Ct})$ and the data were displayed as fold change calculated by the equation of $2-\Delta \Delta \mathrm{Ct}$, in which the $\Delta \Delta \mathrm{Ct}$ was normalized with individual internal control $\mathrm{Ct}$ value.

\subsection{Statistical analysis}

Six mice were used in each group of each time point. All histological and biochemical experiments were performed in triplicate. Data are presented as mean \pm standard error (SE). Statistical analysis was performed using a two-tailed unpaired student's t-test or one-way ANOVA with the Bonferroni method. A p-value $\leq 0.05$ was considered statistically significant.

\section{Results}

3.1 AMD3100 and Maraviroc reduce scar contraction

After removal of the dressings on day 5, grafts were vascularized and adherent. A few of the skin grafts treated with CAS445479-97-0 or Maraviroc were pale and partially ischemic but healed with time. Scars from the mice treated with AMD3100 and Maraviroc were flatter and softer than the control group (Figure 1A). To evaluate wound contraction, wound areas of mice in each group and at each time point were measured using ImageJ software. The wound size of each mouse on Day 0 was set as the original wound area, and wound contraction was displayed as the percentage of wound area calculated by the formula: actual wound area/original wound area*100. Wounds contracted rapidly in the first 4 weeks and had no big changes afterward in the mice of all groups. Significantly less contraction was found in the mice treated with 


\section{International Journal of Medical Science and Health Research}

Vol. 5, No. 02; 2021

ISSN: 2581-3366

AMD3100 at 2 and 4 weeks, and mice treated with Maraviroc at 1 to 5, 7, and 9 weeks, compared to the controls (Figure 1B).

3.2 AMD3100, CAS445479-97-0 and Maraviroc reduce scar thickness and CAS445479-97-0 reduces vascularity

Scars from this mouse model showed similar histologic features of human HTS such as thicker epidermis and dermis and loss of hair follicles, rete ridges, and adnexal structures by H\&E staining. The treatment of chemokine inhibitors improved these histologic features by reducing the dermal thickness and the number of blood vessels in the scars in the whole phase of scarring (Figure 2A). Compared to controls, significantly reduced thickness of the dermis was found in the mice treated with AMD3100 and CAS445479-97-0 at 6 weeks and in the mice treated with Maraviroc at 12 weeks. Vascularity in the scars from the CAS445479-97-0 treated group is significantly lower than controls at 2 weeks (Figure 2B).

3.3 AMD3100 and Maraviroc improve collagen deposition in the scars

Masson's Trichrome stains collagen blue or green. Scars treated with AMD3100 or Maraviroc contained thicker and more loosely arranged collagen fiber in the dermis (Figure 3).

3.4 AMD3100 improves collagen orientation in the dermis of scars

COI was used to evaluate collagen orientation in the scars, ranging from 0 (perfectly random as normal skin) to 1 (perfectly parallel as scars). The COI of scars from the mice treated with AMD3100 was significantly lower at 2 and 6 weeks compared to the controls. However, the COI of scars from the mice treated with CAS445479-97-0 or Maraviroc was significantly higher at 2 weeks (Figure 4).

3.5 AMD3100, CAS445479-97-0 and Maraviroc reduce macrophage recruitment and myofibroblast accumulation

Mouse and human macrophages were identified by F4/80 and CD163 staining, respectively. AMD3100, CAS445479-97-0, and Maraviroc decreased F4/80 (Figure 5A) and CD163 (Figure 5B) positive cell accumulation (brown), especially, compared to controls, AMD3100 significantly decreased F4/80 and CD163 positive cells at all time points. CAS445479-97-0 reduced F4/80 positive cells at 2 weeks. Maraviroc reduced F4/80 cells at 2 and 6 weeks after grafting. CAS445479-97-0 and Maraviroc reduced CD163 positive cells at 2 and 12 weeks after grafting (Figure 5C).

Myofibroblasts were stained for $\alpha$-SMA. All chemokine inhibitors reduced myofibroblast accumulation throughout the whole period. In statistical, AMD3100 significantly decreased the $\alpha$-SMA positive cells at 6 and 12 weeks. CAS445479-97-0 and Maraviroc significantly decreased $\alpha$-SMA positive cells at all time points (Figure 6).

3.6 AMD3100 up-regulates DCN protein expression in the scars

Immunofluorescence staining was performed to investigate DCN level (green) in the scars (Figure 7A). AMD3100 significantly up-regulated DCN in the scars at 2 and 6 weeks compared with controls, but CAS445479-97-0 significantly down-regulated it at 12 weeks, and Maraviroc 


\section{International Journal of Medical Science and Health Research}

Vol. 5, No. 02; 2021

ISSN: 2581-3366

significantly reduced it at 2 and 12 weeks after grafting (Figure 7B). There were no significant differences in serum DCN between treated and control mice (data not shown).

3.7 AMD3100, CAS445479-97-0, and Maraviroc down-regulate fibrotic gene expressions The mRNA level of COL- 1 was found increased, but $\alpha$-SMA and CTGF decreased in the scars from controls gradually over time after grafting. Compared to the control group, AMD3100 significantly reduced COL-1 and HSP47 expression at 12 weeks, $\alpha$-SMA at 6 and 12 weeks, CTGF and TGF- $\beta 1$ at 6 weeks, but increased DCN at 2 weeks after grafting. CAS445479-97-0 increased HSP47 expression at 6 weeks, but reduced COL-1 at 2 and 12 weeks, HSP47 at 12 weeks, $\alpha$-SMA, CTGF, DCN, and TGF- $\beta 1$ at 2 weeks after grafting. Maraviroc reduced COL-1 and $\alpha$-SMA expression at 2 and 12 weeks, HSP47 at 12 weeks, CTGF, DCN, and TGF- $\beta 1$ at 2 weeks, but increased HSP47 at 6 weeks after grafting (Figure 8).

\section{Discussions}

In this study, we blocked SDF-1/CXCR4, MCP-1/CCR2, and CCL5/CCR5 pathways by AMD3100, CAS445479-97-0, and Maraviroc to evaluate scar formation using a dermal scar mouse model. The inhibitors of these chemokine pathways significantly improved scar features. Macrophages arise from bloodborne cells before accumulating in wounds and have important roles in wound healing processes such as directing fibroblast proliferation, myofibroblast differentiation, and collagen deposition in wounds (28). However, increased numbers and activity of macrophages could lead to HTS formation with excessive ECM synthesis. In a dermal scar mouse model, the loss of macrophages in the subacute phase of wound healing decreased $\operatorname{HTS}$ formation $(9,17,18,29)$. In normal wound healing, myofibroblasts are responsible for both tissue contraction and the secretion of ECM and undergo apoptosis at the end of the healing process. Significant quantities of ECM components, such as type I and III collagen, fibronectin, laminin, and other basal membrane proteins, are synthesized by myofibroblasts. The persistent presence and activity of myofibroblasts characterize pathological scars such as HTS or keloids (30-31). The decreased numbers of macrophages and myofibroblasts in scars treated with these chemokine pathway inhibitors might contribute to reduce scarring and contracture in transplanted human skin in vivo.

Collagen is the main component of ECM, and collagen bundles are organized in a threedimensional random basket-weave pattern in normal skin. Compared with normal skin, the collagen bundles in HTS and keloids are organized in a more parallel pattern. COI, ranging from 0 to 1 , is lower in normal skin and mature scars than pathological scars, such as HTS and keloids (32-33). AMD3100 treated mice had a lower COI and thus more random orientated collagen bundles typical of more normal or mature scar compared to the control group.

Fibroblasts synthesize and secrete ECM content such as collagen I and CTGF, and then regulate ECM metabolism by releasing various cytokines, including TGF- $\beta 1$ and matrix metalloproteinases. Abnormal ECM reconstruction contributes to HTS (10, 34). Up-regulated COL-I mRNA levels represent increased collagen deposition, which is one of the primary characteristics of human HTS (35). CTGF promotes cell proliferation, migration, differentiation, angiogenesis, ECM production, and adhesion. In wounded skin, CTGF mRNA levels are upregulated and suppression of CTGF decreased the severity of scar formation (36). In a dermal 


\section{International Journal of Medical Science and Health Research}

Vol. 5, No. 02; 2021

ISSN: 2581-3366

scar mouse model, HSP47 play an important role in collagen biosynthesis and up-regulated HSP47 mRNA levels consistent with the increased synthesis and the altered assembly of collagen during scar formation (17). TGF- $\beta$ is a crucial factor in the regulation of contraction in normal skin fibroblasts and promotes fibroblast chemotaxis to the inflammatory site to generate ECM proteins. The transformation from fibroblasts to myofibroblasts is impaired by TGF- $\beta 1$, which is a profibrotic mediator and regulator of wound healing cellular processes such as proliferation, differentiation, migration, cell survival, and angiogenesis. Dysregulation of production or activity of TGF- $\beta$ can cause abnormal fibrosis (10). Blocking SDF-1/CXCR4, MCP-1/CCR2, and CCL5/CCR5 pathways reduced these fibrotic features and contributed to the improvement of collagen deposition and scar formation in a dermal scar mouse model.

DCN is a small leucine-rich proteoglycan and regulates collagen fibrillogenesis by binding type I collagen and inhibiting fibrogenic activity of TGF- $\beta 1$. Decorin and fibromodulin have been found more in the superficial layers than in the deeper dermal layers in normal skin. The expression of biglycan, fibromodulin, and lumican are increased in the basement membrane and around basal epithelial cells. Compared with normal skin, these proteoglycans are absent or weakly expressed in HTS (37-38). DCN is significantly downregulated in HTS tissue and blocking microRNA $181 \mathrm{~b}$ to increase DCN has been reported to be a potential therapy for HTS (39). Blocking SDF-1/CXCR4 pathway increased DCN level in the scars and significantly reduced TGF- $\beta$. Up-regulated DCN may have contributed to reduced hypertrophic scarring. Based on our findings, blocking SDF-1/CXCR4, MCP-1/CCR2, and CCL5/CCR5 pathways significantly improved scarring in the dermal scar mouse model. Via coagulation, leukocyte infiltration, re-epithelialization, angiogenesis, stem cell recruitment, collagen re-organization, and lymphocyte-mediated immune response, chemokines are fundamental for wound healing and scar formation. Blood-borne cells are progenitor cells of macrophages and fibrocytes, and the recruitment of these cell to the wound sites from systemic circulation after dermal injury is regulated by chemokines such as SDF-1 released from dermal fibroblasts. They contribute to not only wound healing but also hypertrophic scarring.

To regulate these processes, inhibition of chemokine pathways such as SDF-1/CXCR4, MCP1/CCR2, and CCL5/CCR5 has potential to reduce hypertrophic scarring and offers therapeutic strategies for HTS.
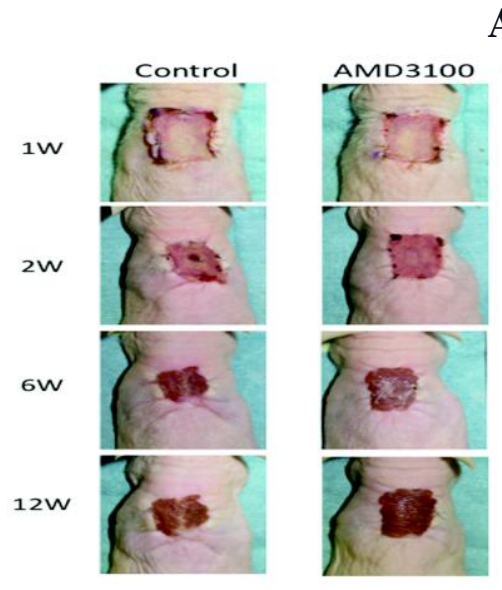

A
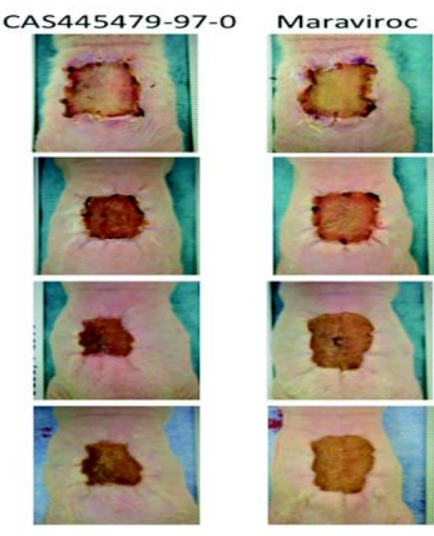


\section{B}

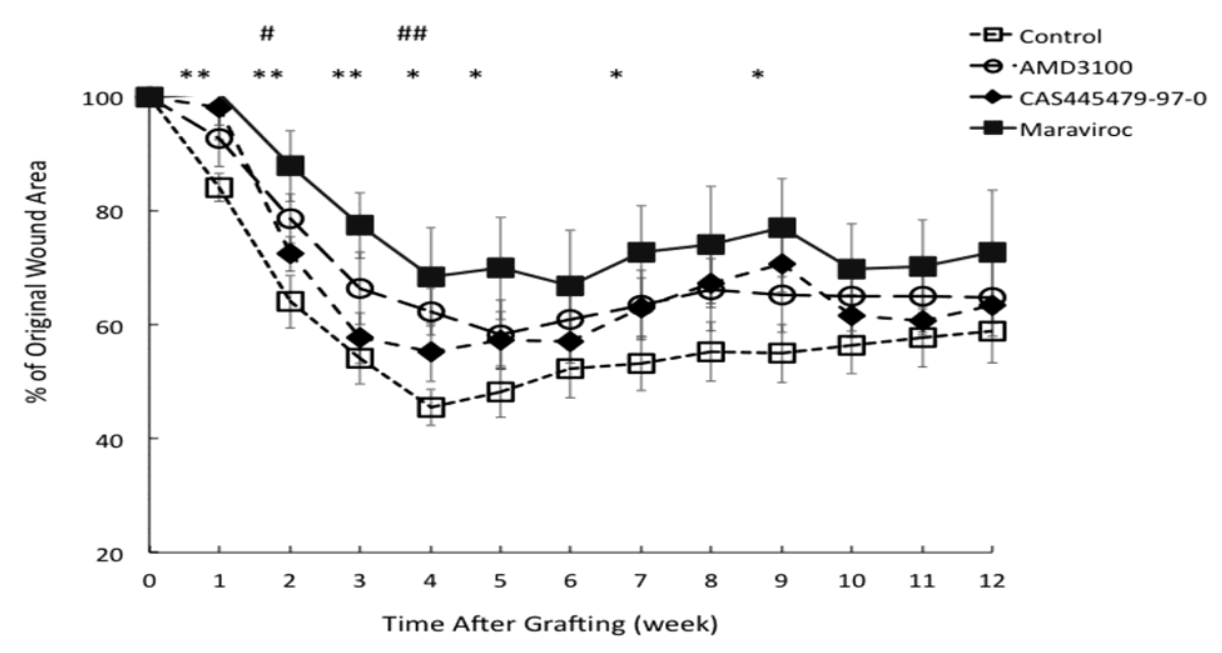

Figure 1. Human split-thickness skin grafts develop scars in athymic nude mice. (A) Representative images of scars in the mice treated with AMD3100, CAS445479-97-0, Maraviroc, or vehicle (control) at 1, 2, 6, and 12 weeks (W) after human skin grafting into nude mice. (B) Wound area was measured in Image J. Wound contraction was assessed relative to the original wound and displayed as \% of Original Wound Area. N=6; \# AMD3100 vs Control, * Maraviroc vs Control; \# or * $\mathrm{p} \leq 0.05$; \#\# or $* * \mathrm{p} \leq 0.01$.

A

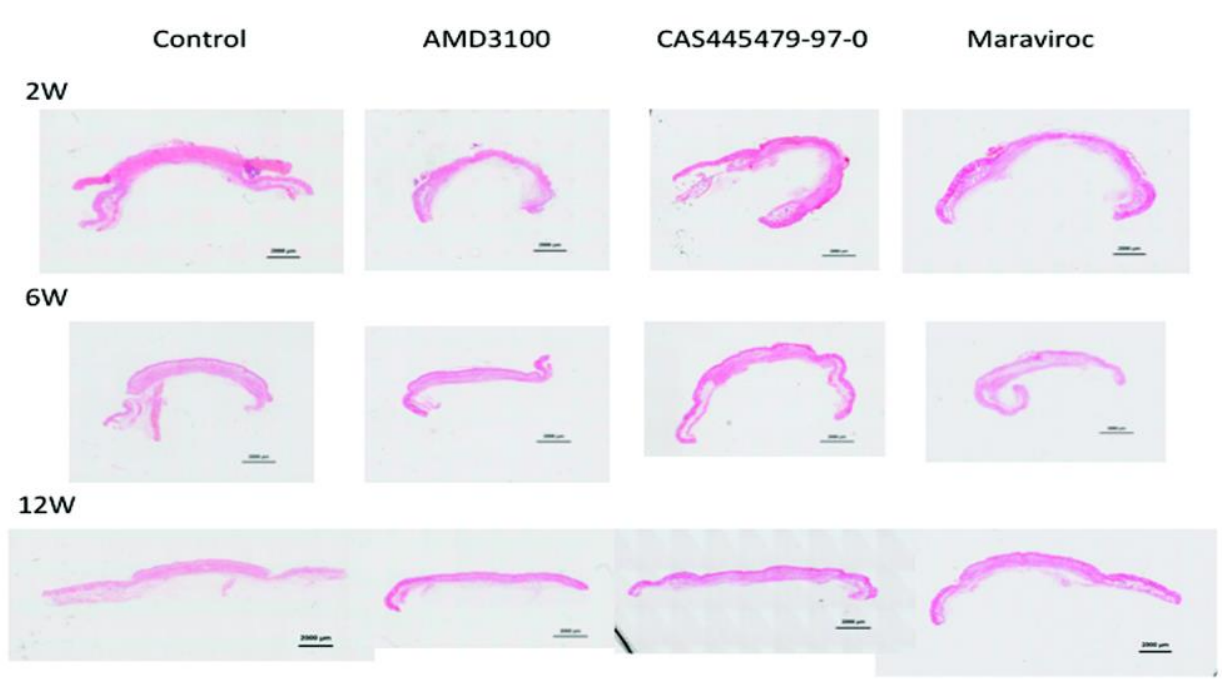


Vol. 5, No. 02; 2021

ISSN: 2581-3366

\section{B}
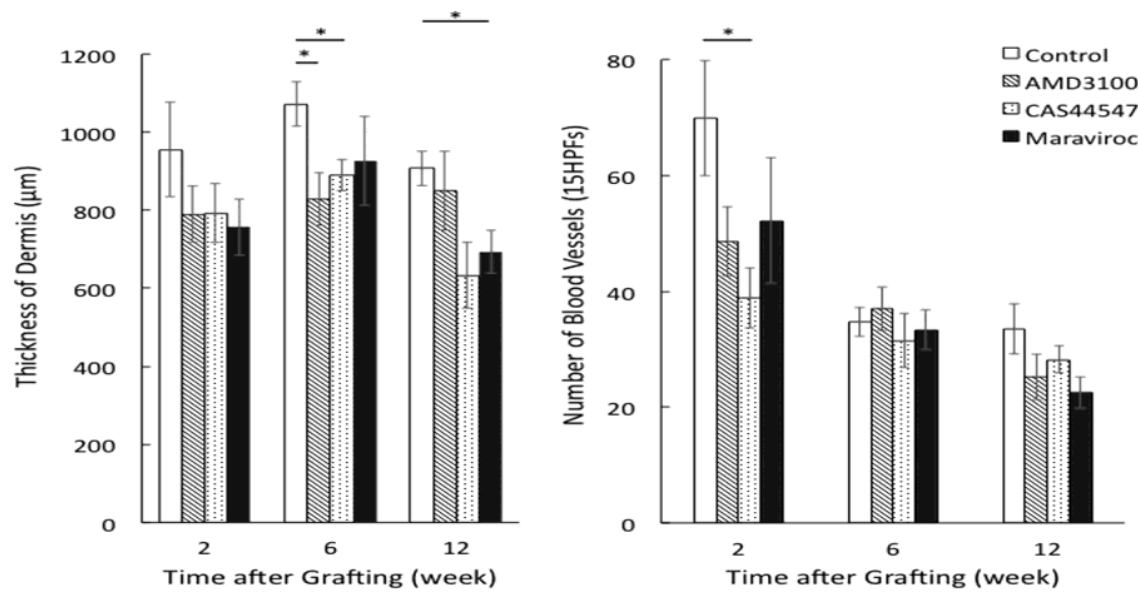

Figure 2. Scar thickness and vascularity in the dermis of scars. (A) Paraffin-embedded scar sections were stained by Hematoxylin and Eosin (H\&E), which includes the scars and mouse skin on both sides collected from the mice treated with AMD3100, CAS445479-97-0, Maraviroc, or vehicle at 2, 6, and 12 weeks after grafting. Scale bar in each slide represents 2000 $\mu \mathrm{m}$. (B) The dermal thickness was measured at three locations as described in the Method section, and the mean values are displayed as $\mu \mathrm{m}$. The numbers of blood vessels were counted in the dermis of scars in 15 high-power fields (HPFs). N=6; * $\mathrm{p} \leq 0.05$.

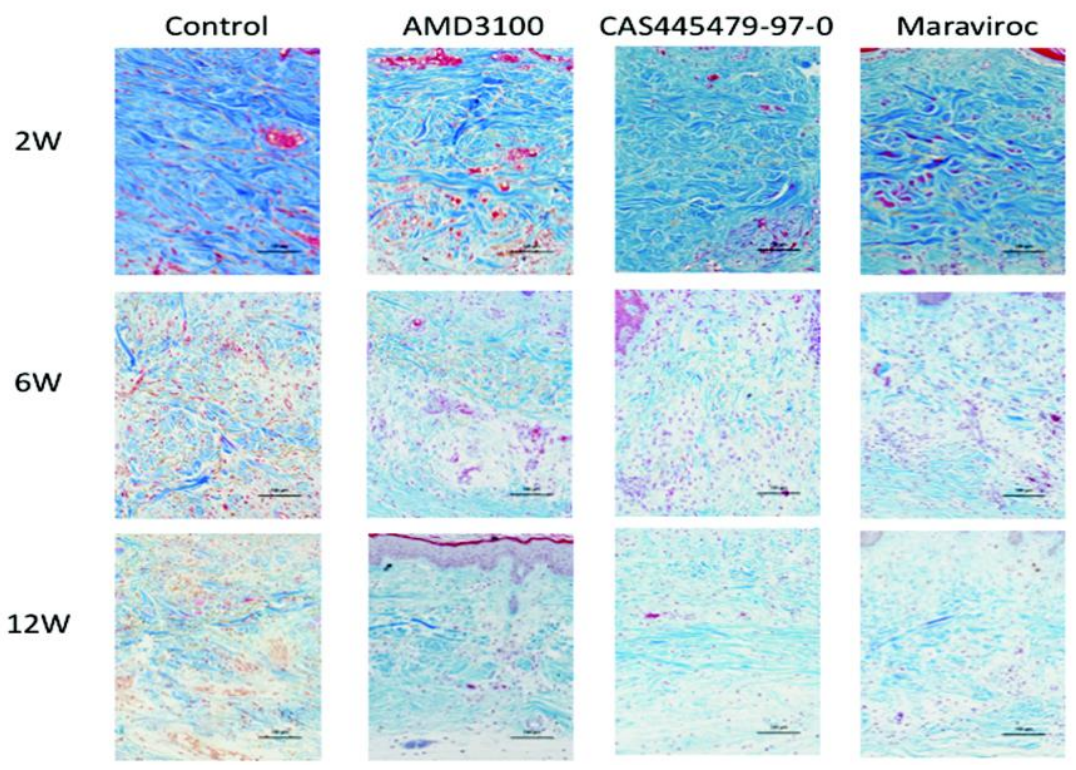

Figure 3. Morphology of collagen fibers in the scars. Representative Masson's trichrome images of collagen fibers in the scars from the mice treated with AMD3100, CAS445479-97-0, 
Vol. 5, No. 02; 2021

ISSN: 2581-3366

Maraviroc, or vehicle at 2, 6, and 12 weeks after grafting, which stains collagen blue, keratin red, and cell nuclei black. Scale bar in each slide is $100 \mu \mathrm{m}$.

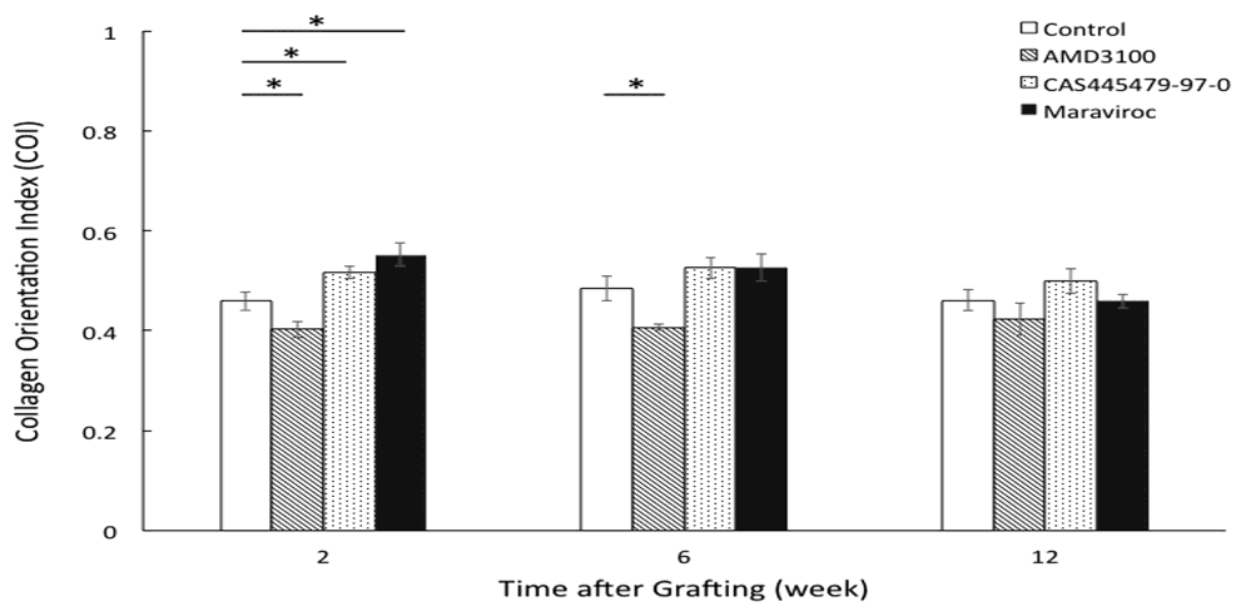

Figure 4. Collagen orientation index (COI). Collagen fibers were stained by Picrosirus Red in the scars from the mice treated with AMD3100, CAS445479-97-0, Maraviroc, or vehicle at 2, 6, and 12 weeks after grafting. Collagen bundle structure is evaluated as COI. $\mathrm{N}=6 ;{ }^{*} \mathrm{p} \leq 0.05$.

A

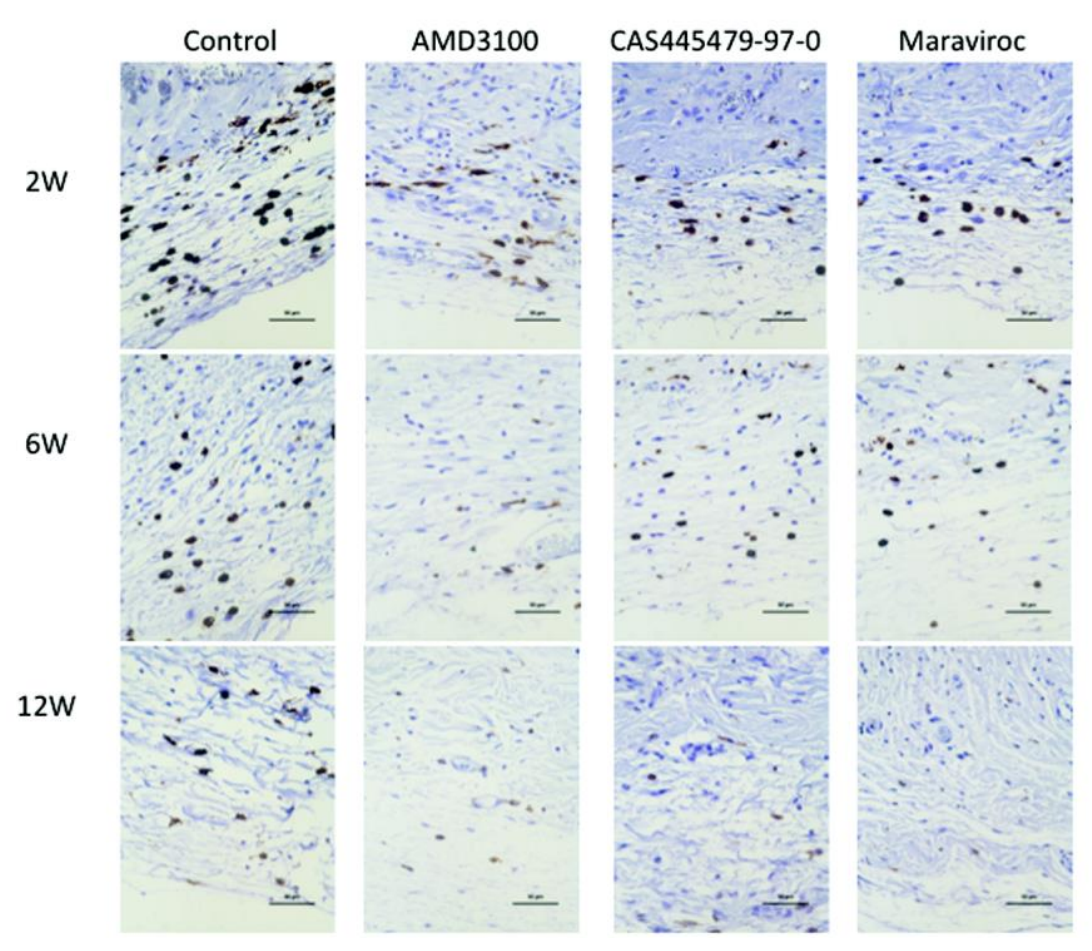



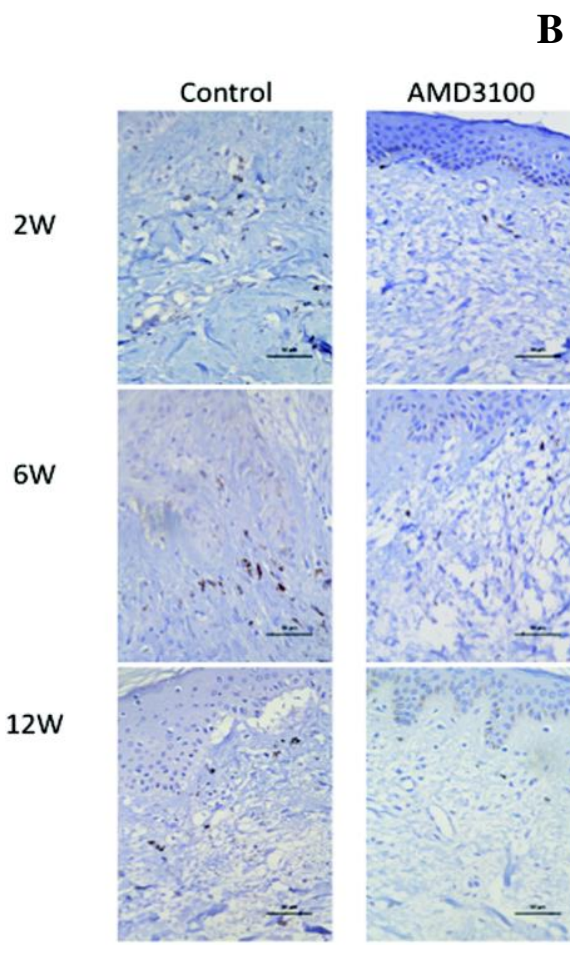

B
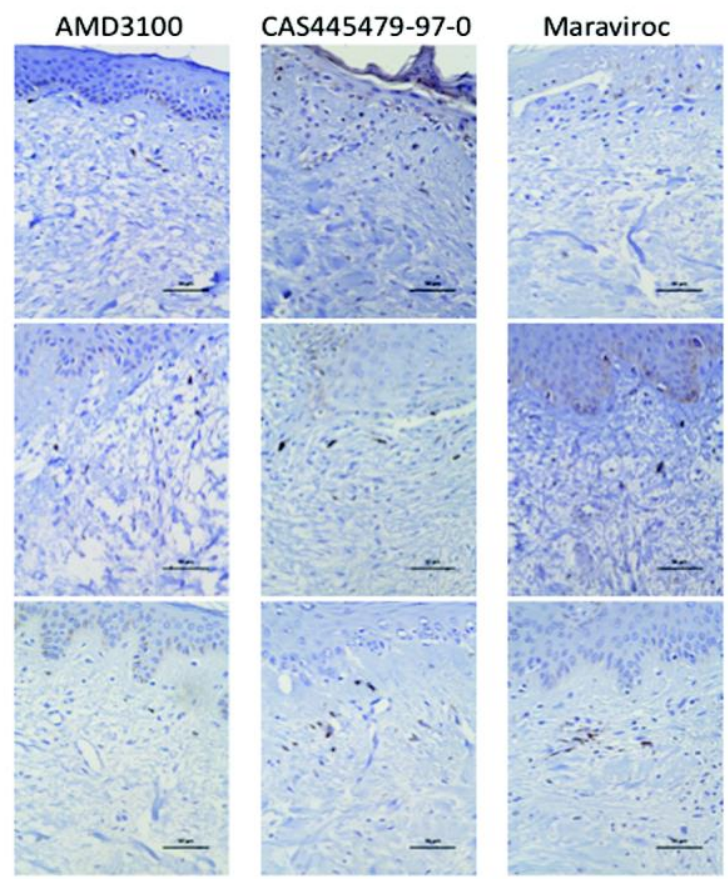

C

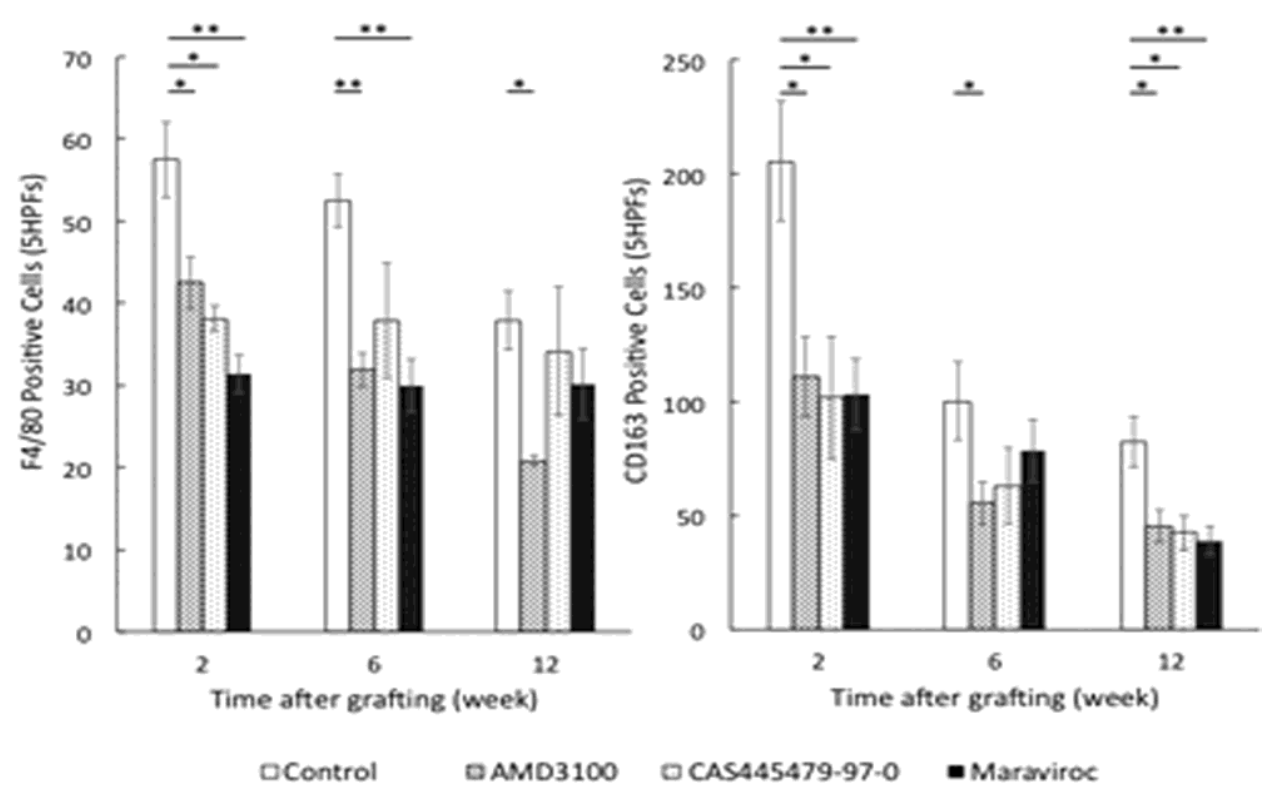

Figure 5. Human and mouse macrophages in the scars. Scar sections were stained for F4/80 and CD163 using the avidin-biotin complex (ABC) method. (A-B) Representative images of F4/80 and CD163 staining in the scars from the mice treated with AMD3100, CAS445479-97-0, 
Maraviroc, or vehicle at 2, 6, and 12 weeks after grafting. Scale bar in each slide is $50 \mu \mathrm{m}$. (C) The total F4/80- and CD163-expressing cells were counted in five HPFs of the dermis. N=6; * $\mathrm{p} \leq 0.05, * * \mathrm{p} \leq 0.01$.

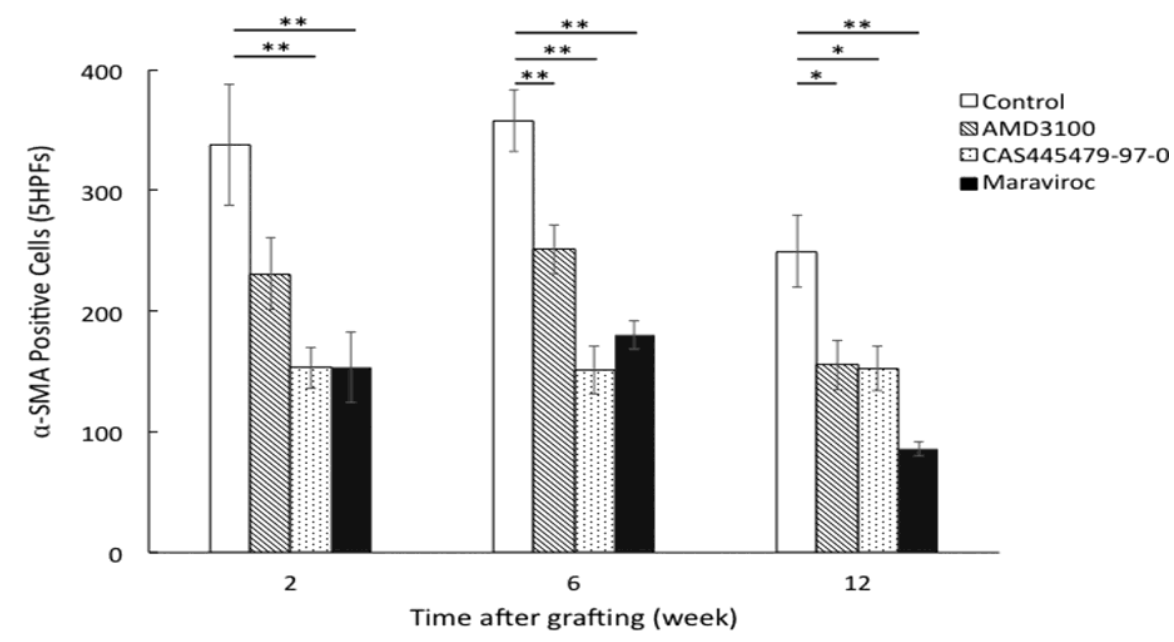

Figure 6. Myofibroblast accumulation in the scars. $\square$ Scar sections were stained for $\alpha$-SMA using the avidin-biotin complex (ABC) method, which were collected from the mice treated with AMD3100, CAS445479-97-0, Maraviroc, or vehicle at 2, 6, and 12 weeks after grafting. $\mathrm{N}=6$; * $\mathrm{p} \leq 0.05 ; * * \mathrm{p} \leq 0.01 . \alpha-\mathrm{SMA}$, alpha-smooth muscle actin.

A

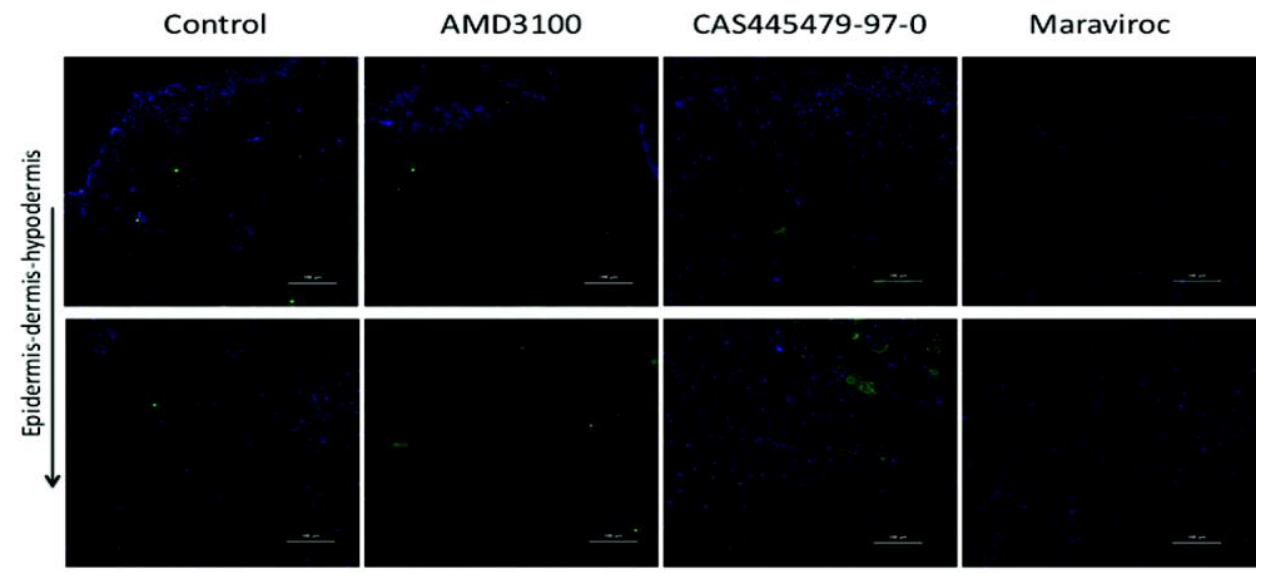

Blue: DAPI

Green: DCN 
Vol. 5, No. 02; 2021

ISSN: 2581-3366

\section{B}

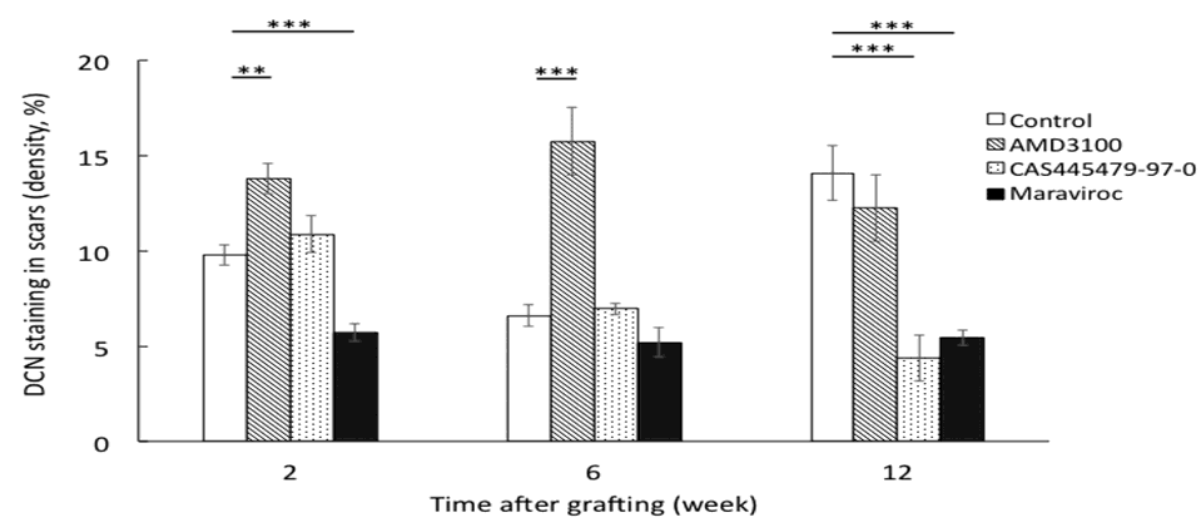

Figure 7. DCN expression in the scars. Scar sections were stained for DCN and DAPI using Immunofluorescence. (A) Representative images of DCN (green) and DAPI (blue) in the scars from the mice treated with AMD3100, CAS445479-97-0, Maraviroc, or vehicle at 2 weeks after grafting. Scale bar in each slide is $100 \mu \mathrm{m}$. (B) The density of DCN staining was calculated in each scar section using ImageJ software. $\mathrm{N}=6 ;{ }^{* *} \mathrm{p} \leq 0.01, * * * \mathrm{p} \leq 0.001$; DCN, decorin.
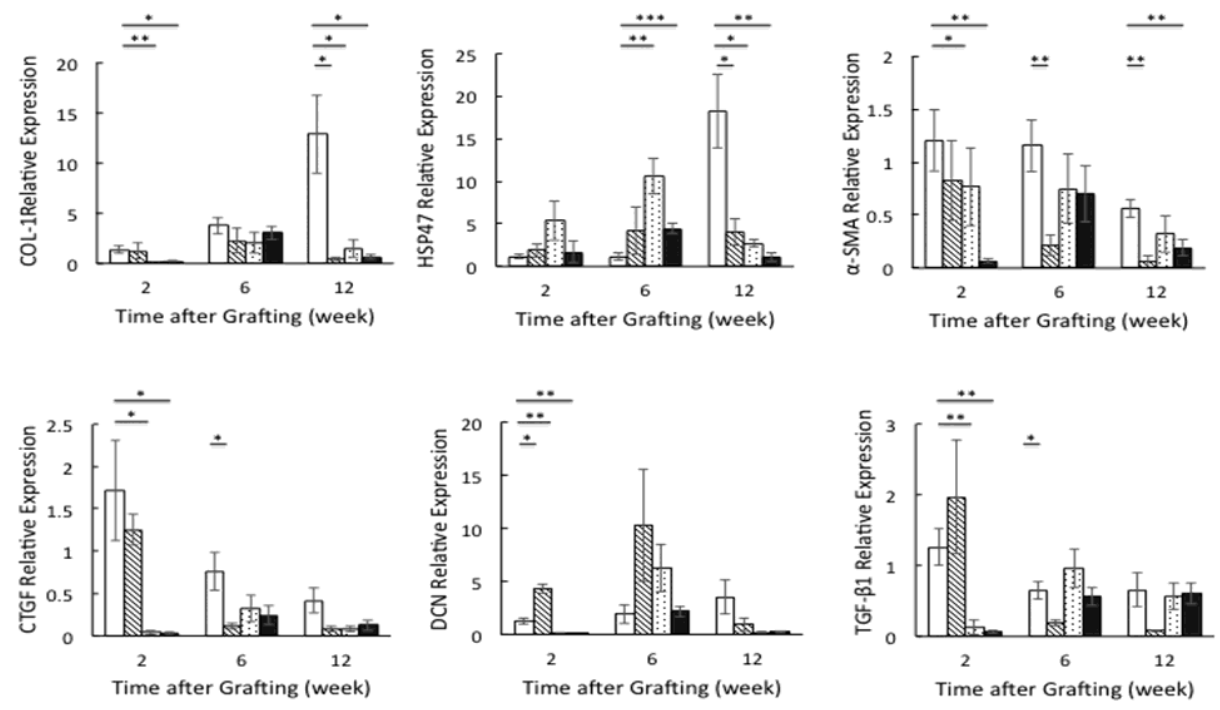

$\square$ Control

$\triangle A M D 3100$

ECAS445479-97-0

- Maraviroc

Figure 8. mRNA level of COL-1, HSP47, $\alpha$-SMA, CTGF, DCN, and TGF- $\beta 1$ genes in the scars. Scar biopsies collected from the mice treated with AMD3100, CAS445479-97-0, Maraviroc, or vehicle were powdered and then lysed for total RNA extraction and cDNA synthesis. The mRNA levels of COL-1, HSP47, $\alpha$-SMA, CTGF, DCN, and TGF- $\beta 1$ were quantified by realtime $\mathrm{qPCR} . \mathrm{N}=6 ; * \mathrm{p} \leq 0.05, * * \mathrm{p} \leq 0.01,{ }^{* * *} \mathrm{p} \leq 0.001$; COL-1, type 1 collagen; HSP47, heat shock 


\section{International Journal of Medical Science and Health Research}

Vol. 5, No. 02; 2021

ISSN: 2581-3366

protein 47; $\alpha$-SMA, alpha-smooth muscle actin; CTGF, connective tissue growth factor; DCN, decorin; TGF- $\beta 1$, transforming growth factor beta 1 .

\section{Acknowledgments}

University Hospital Foundation from University of Alberta and the Firefighters' Burn Trust Fund from Edmonton Firefighter's Burn Treatment Society supported this work.

\section{References}

1. Zhu Z, Ding J, Tredget EE. The molecular basis of hypertrophic scars. Burns Trauma. $2016 ; 4: 2$

2. Alrobaiea SM, Ding J, Ma ZS, Tredget EE. A novel nude mouse model of hypertrophic scarring using scratched full thickness human skin grafts. Adv Wound Care (New Rochelle). 2016;5(7):299-313.

3. Sriram G, Bigliardi PL, Bigliardi-Qi M. Fibroblast heterogeneity and its implications for engineering organotypic skin models in vitro. Eur J Cell Biol. 2015;94(11):483-512.

4. Rees PA, Greaves NS, Baguneid M, Bayat A. Chemokines in Wound Healing and as Potential Therapeutic Targets for Reducing Cutaneous Scarring. Adv Wound Care (New Rochelle). 2015 Nov 1;4(11):687-703.

5. Ding J, Tredget EE. The Role of Chemokines in Fibrotic Wound Healing. Adv Wound Care (New Rochelle). 2015 Nov 1;4(11):673-686.

6. Martins-Green M, Petreaca M, Wang L. Chemokines and their receptors are key players in the orchestra that regulates wound healing. Adv Wound Care (New Rochelle) 2013;2: 327347.

7. Manuela Martins-Green, Melissa Petreaca, Lei Wang. Chemokines and Their Receptors Are Key Players in the Orchestra That Regulates Wound Healing. Adv Wound Care (New Rochelle) 2013 Sep; 2(7): 327-347.

8. Ding J, Hori K, Zhang R, Marcoux Y, Honardoust D, Shankowsky HA, Tredget EE. Stromal cell-derived factor 1 (SDF-1) and its receptor CXCR4 in the formation of postburn hypertrophic scar (HTS). Wound Repair Regen. 2011 Sep-Oct;19(5):568-78.

9. Ding J, Ma Z, Liu H, Kwan P, Iwashina T, Shankowsky HA, Wong D, Tredget EE. The therapeutic potential of a C-X-C chemokine receptor type 4 (CXCR-4) antagonist on hypertrophic scarring in vivo. Wound Repair Regen. 2014 Sep-Oct;22(5):622-30.

10. Hirokatsu U, Ding J, Tredget EE. Chemokine pathway can be the potential therapeutic target for hypertrophic scar. EMJ. 2019;4[2]:55-63.

11. Yadav A, Saini V, Arora S. MCP-1: chemoattractant with a role beyond immunity: a review. Clin Chim Acta. 2010 Nov 11;411(21-22):1570-9.

12. Brancato SK, Albina JE. Wound macrophages as key regulators of repair: origin, phenotype, and function. Am J Pathol 2011;178:19-25. 


\section{International Journal of Medical Science and Health Research}

Vol. 5, No. 02; 2021

ISSN: 2581-3366

13. Raman D, Sobolik-Delmaire T, Richmond A. Chemokines in health and disease. Exp Cell Res 2011;317:575-589.

14. Wong VW, Rustad KC, Akaishi S, Sorkin M, Glotzbach JP, Januszyk M, Nelson ER, Levi K, Paterno J, Vial IN, Kuang AA, Longaker MT, Gurtner GC. Focal adhesion kinase links mechanical force to skin fibrosis via inflammatory signaling. Nat Med 2011;18:148-152.

15. Wang SW, Liu SC, Sun HL, Huang TY, Chan CH, Yang CY, Yeh HI, Huang YL, Chou WY, Lin YM, Tang CH. CCL5/CCR5 axis induces vascular endothelial growth factormediated tumor angiogenesis in human osteosarcoma microenvironment. Carcinogenesis. 2015 Jan;36(1):104-14.

16. Ishida Y, Kimura A, Kuninaka Y, Inui M, Matsushima K, Mukaida N, Kondo T. Pivotal role of the CCL5/CCR5 interaction for recruitment of endothelial progenitor cells in mouse wound healing. J Clin Invest 2012;122:711-721.

17. Wang J, Ding J, Jiao H, Honardoust D, Momtazi M, Shankowsky HA, Tredget EE. Human hypertrophic scar-like nude mouse model: characterization of the molecular and cellular biology of the scar process. Wound Repair Regen 2011; 19: 274-85.

18. Momtazi M, Kwan P, Ding J, Anderson CC, Honardoust D, Goekjian S, Tredget EE. A nude mouse model of hypertrophic scar shows morphologic and histologic characteristics of human hypertrophic scar. Wound Repair Regen 2013; 21: 77-87.

19. Nishimura Y, Ii M, Qin G, Hamada H, Asai J, Takenaka H, Sekiguchi H, Renault MA, Jujo K, Katoh N, Kishimoto S, Ito A, Kamide C, Kenny J, Millay M, Misener S, Thorne T, Losordo DW. CXCR4 antagonist AMD3100 accelerates impaired wound healing in diabetic mice. J Invest Dermatol. 2012 Mar;132(3 Pt 1):711-20. doi: 10.1038/jid.2011.356. Epub 2011 Nov 3.

20. Chu PY, Walder K, Horlock D, Williams D, Nelson E, Byrne M, Jandeleit-Dahm K, Zimmet P, Kaye DM. CXCR4 Antagonism Attenuates the Development of Diabetic Cardiac Fibrosis. PLoS One. 2015 Jul 27;10(7).

21. Liu J, Xue Y, Dong D, Xiao C, Lin C, Wang H, Song F, Fu T, Wang Z, Chen J, Pan H, Li Y, Cai D, Li Z. CCR2- and CCR2+ corneal macrophages exhibit distinct characteristics and balance inflammatory responses after epithelial abrasion. Mucosal Immunol. 2017 Sep;10(5):1145-1159.

22. Mencarelli A, Graziosi L, Renga B, Cipriani S, D'Amore C, Francisci D, Bruno A, Baldelli F, Donini A, Fiorucci S. CCR5 Antagonism by Maraviroc Reduces the Potential for Gastric Cancer Cell Dissemination. Transl Oncol. 2013 Dec 1;6(6):784-93.

23. De Clercq E. Mozobil (Plerixafor, AMD3100), 10 years after its approval by the US Food and Drug Administration. Antivir Chem Chemother. 2019 JanDec;27:2040206619829382. 


\section{International Journal of Medical Science and Health Research}

Vol. 5, No. 02; 2021

ISSN: 2581-3366

24. Cherney RJ, Mo R, Meyer DT, Pechulis AD, Guaciaro MA, Lo YC, Yang G, Miller PB, Scherle PA, Zhao Q, Cvijic ME, Barrish JC, Decicco CP, Carter PH. Benzimidazoles as benzamide replacements within cyclohexane-based CC chemokine receptor 2 (CCR2) antagonists. Bioorg Med Chem Lett. 2012 Oct 1;22(19):6181-4.

25. Martin-Blondel G, Brassat D, Bauer J, Lassmann H, Liblau RS. CCR5 blockade for neuroinflammatory diseases--beyond control of HIV. Nat Rev Neurol. 2016 Feb;12(2):95-105. Review.

26. Verhaegen PD, Schouten HJ, Tigchelaar-Gutter W, van Marle J, van Noorden CJ, Middelkoop E, van Zuijlen PP. Adaptation of the dermal collagen structure of human skin and scar tissue in response to stretch: an experimental study. Wound Repair Regen. 2012 Sep-Oct;20 (5):658-66.

27. Van Zuijlen PP1, Ruurda JJ, van Veen HA, van Marle J, van Trier AJ, Groenevelt F, Kreis RW, Middelkoop E. Collagen morphology in human skin and scar tissue: no adaptations in response to mechanical loading at joints. Burns. 2003 Aug;29(5):423-31.

28. Sinha M, Sen CK, Singh K, Das A, Ghatak S, Rhea B, Blackstone B, Powell HM, Khanna S, Roy S. Direct conversion of injury-site myeloid cells to fibroblast-like cells of granulation tissue. Nat Commun. 2018 Mar 5;9(1):936.

29. Li-Tsang C, Feng BB, Huang L, Liu XH, Shu B, Chan YTY, Cheung KK. A histological study on the effect of pressure therapy on the activities of myofibroblasts and keratinocytes in hypertrophic scar tissues after burn. Burns. 2015;41(5):1008-16.

30. Darby IA, Zakuan N, Billet F, Desmoulière A. The myofibroblast, a key cell in normal and pathological tissue repair. Cell Mol Life Sci. 2016 Mar;73(6):1145-57.

31. Sarrazy V, Billet F, Micallef L, Coulomb B, Desmoulière A. Mechanisms of pathological scarring: role of myofibroblasts and current developments. Wound Repair Regen. 2011 Sep;19 Suppl 1:s10-5.

32. Verhaegen PD, van Zuijlen PP, Pennings NM, van Marle J, Niessen FB, van der Horst CM, Middelkoop E. Differences in collagen architecture between keloid, hypertrophic scar, normotrophic scar, and normal skin: An objective histopathological analysis. Wound Repair Regen. 2009 Sep-Oct;17(5):649-56.

33. Westra I, Verhaegen PDHM, Ibrahim Korkmaz H, Braam KI, Kaspers GJL, Niessen HWM, Niessen FB. Investigating histological aspects of scars in children. J Wound Care. 2017 May 2;26(5):256-265.

34. Liu F, Chen WW, Li Y, Zhang JQ, Zheng QB. MiR-6836-3p promotes proliferation of hypertrophic scar fibroblasts by targeting CTGF. Eur Rev Med Pharmacol Sci. 2018 Jul;22(13):4069-4074. 
Vol. 5, No. 02; 2021

ISSN: 2581-3366

35. Tredget EE, Nedelec B, Scott PG, Ghahary A. Hypertrophic scars, keloids, and contractures. The cellular and molecular basis for therapy. Surg Clin North Am. 1997 Jun;77(3):70130.

36. Jensen J, Gentzkow G, Berman G, Senne L, Jewell M, Connall TP, Miller SR, Galiano RD, Young L. Anti-CTGF Oligonucleotide Reduces Severity of Postsurgical Hypertrophic Scars in a Randomized, Double-Blind, Within-Subject, Placebo-Controlled Study. Plast Reconstr Surg. 2018 Aug;142(2):192e-201e.

37. Honardoust D, Varkey M, Marcoux Y, Shankowsky HA, Tredget EE. Reduced Decorin, Fibromodulin and TGF-beta3 in Deep Dermis Leads to Hypertrophic Scar. J Burn Care Res. 2012; 33(2):218-27. doi:10.1097/BCR.0b013e3182335980 PMID: 22079916

38. Darby IA, Laverdet B, Bonté F, Desmoulière A. Fibroblasts and myofibroblasts in wound healing. Clin Cosmet Investig Dermatol. 2014 Nov 6;7:301-11.

39. Kwan P, Ding J, Tredget EE. MicroRNA 181b regulates decorin production by dermal fibroblasts and may be a potential therapy for hypertrophic scar. PLoS One. 2015 Apr $2 ; 10(4)$. 